\title{
Energy Reduction in Mobile WSN using Dynamic Clustering \& M-Leach Routing Approach
}

\author{
Mohd. Yasser $^{1}$, Manjot Kaur ${ }^{2}$ \\ ${ }^{1}$ Research Scholar, Department of Computer Science \& Engineering, Chandigarh Group of Colleges, Gharuan \\ ${ }^{2}$ Associate Professor, Department of Computer Science \& Engineering, C.G.C, Gharuan
}

\begin{abstract}
Wireless sensor network is used for sensing the information from that area which is out of human range. In the mobile wireless sensor network nodes are available in mobility mode. The sensor nodes sense the information by moving from one destination to second. In the whole processing of sensing and transmitting data energy has been consumed. In the purposed work the energy utilization has been reduced by using clustering approach that increase the network life time. In this work mobile leach approach has been utilized for development of clusters in the network. Cluster head selection has been done on the basis of maximum energy. To recover the data recovery node has been initialized that sense the information at an interval of time so that data can be recovered from recovery node at time of failure of any node. On the basis of different parameters performance has been evaluated.
\end{abstract}

Keywords: WSN, MWSN, Clustering, Routing, AODV.

\section{Introduction}

\subsection{Wireless Sensor Network}

WSN networks compose possibly large number of wireless sensor nodes that are resource constrained in terms of energy, memory, computing capabilities and communication strange [1]. A sensor node will be also referred to as just node or sensor in the sequel. There are various type of application of WSN are already present in health care, navigation, rescue, intelligent transportation, social networking, gaming application fields, and critical infrastructure protection [2]. This network is of tenant attended and deployed in harsh environments. WSNs are hence subject to several threats because of their nature [3].

The WSN is built of "nodes" - from a few to several hundreds or even thousands, where each node is connected to one (or sometimes several) sensors. Each such sensor network node has typically several parts [4]. In this every network node contain a radio transceiver with internal or external antenna connection, a microcontroller an electronic circuit for interfacing with the sensors and an energy source usually a battery or an embedded form of energy harvesting. A sensor node might vary in size from that of a shoebox down to the size of a grain of dust, although functioning [5] "motes" of genuine microscopic dimensions have yet to be created [6].

\subsection{MWSN}

Mobile wireless sensor networks (MWSNs) can simply be defined as a wireless sensor network (WSN) in which the sensor nodes are mobile [7]. MWSNs are a smaller, emerging field of research in contrast to their wellestablished predecessor [8]. MWSNs are much more versatile than static sensor networks as they can be deployed in any scenario and cope with rapid topology changes [9]. However, many of their applications are similar, such as environment monitoring or surveillance commonly the nodes consist of a radio transceiver and a microcontroller powered by a battery [10]. As well as some kind of sensor for detecting light, heat, humidity, temperature, etc.

\subsection{Energy optimization in MWSN}

A typical Mobile wireless sensor network consists of sensors powered by small batteries that are difficult to replace if not impossible. Hence, the sensor nodes can only transmit a finite number of bits before they run out of energy [11]. Thus, reducing the energy consumption per bit for end-toend data transmission is an important design consideration for such networks. It assume that each information bit collected by a sensor is useful for a finite amount of time; after this time the information may become irrelevant. Hence all the bits collected by the sensors need to be communicated to a hub node before a certain deadline [12]

Mobile Wireless sensor networks typically have power constraints. The absence of wires implies the lack of an external power supply such as battery packs. Although photovoltaic or other passive energy gathering techniques are possible, these approaches typically provide only a modest amount of operating power. Therefore it is necessary to extend the battery life individual sensors so that the network can remain functional as long as possible. Moreover, for biomedical sensors, power usage results in heat dissipation that may further require minimizing the total power consumed by the wireless sensor network [13].

\subsection{Clustering in MWSN}

Cluster-based energy balancing scheme is intended to ameliorate the above energy unbalancing phenomena. We exploit the observation that in a heterogeneous sensor network there are nodes which are more powerful in terms of energy reserve and wireless communication ability [14]. We transform the flat communication infrastructure into a hierarchical one where strong nodes act as cluster heads to gather information within the clusters and then communicate with the sink directly via single hop link. In such a way, the hot spot around the sink is divided into multiple regions around the cluster heads in the hierarchical infrastructure. 


\section{International Journal of Science and Research (IJSR) \\ ISSN (Online): 2319-7064 \\ Index Copernicus Value (2013): 6.14 | Impact Factor (2014): 5.611}

These distributed regions will assume fewer burdens due to the smaller scale of sensor nodes within the clusters. Wireless sensor networks are composed by a large amount of small, resource constrained devices, called sensor nodes, which have limited sensing, computing and wireless communication abilities. These sensor nodes usually collaborate with each other via multi-hop links. The member node takes care of the transmission and arrangement of nodes within the cluster [15]. The cluster head takes care of transferring the data to other clusters within the network by maintaining the routing information. The member nodes report their data to the respective $\mathrm{CHs}$. The $\mathrm{CHs}$ aggregate the data and send them to the central base through other CHs. Because CHs often transmit data over longer distances, they lose more energy compared to member nodes. The network may be re-clustered periodically in order to select energy-abundant nodes to serve as $\mathrm{CHs}$, thus distributing the load uniformly on all the nodes.

\subsection{Routing Protocols in WSN}

We can reduce the energy consumption by using various techniques like data aggregation, clustering, data-centric methods, etc. The routing protocols can be classified as flat, hierarchical or location-based as follow [16]:

\subsubsection{Flat networks}

In this network equal nodes are used. Hence each node plays the same role. This network has no logical hierarchy. It uses a flat addressing scheme. The example of flat network is Routing Information Protocol (RIP).

\subsubsection{Hierarchical networks}

The nodes are partitioned into a number of small groups called clusters. Each cluster has a cluster head $(\mathrm{CH})$ which is the coordinator of other nodes. These $\mathrm{CHs}$ perform data aggregation so that energy inefficiency may be reduced. The cluster heads may change. The node which has the highest energy acts as the $\mathrm{CH}$. Hierarchical routing is an efficient way to lower energy consumption within a cluster. It has major advantages of scalability, energy efficiency, efficient bandwidth utilization, reduces channel contention and packet collisions [17].

\subsubsection{Location-based networks}

In location-based clustering, the location of the sensor nodes plays a important role. Base station is used to send data to a particular location. In these protocols, the awareness of position of the sensor nodes is very significant to transfer the data to destinations. The distance between neighboring nodes can be estimated on the basis of incoming signal strengths. On the basis of location based protocol, if there is no activity then nodes should go to sleep to save energy [18].

\section{Related Work}

Muhammad Arshad1 et al [13] "Efficient Cluster Head Selection Scheme in Mobile Data Collector Based Routing Protocol" describes Mobile Wireless Sensor Network (MWSN) is one of the rising and emerging technologies for various application of NWGN. The enormous concerns of these networks are energy efficiency and data aggregation within the network. The aim of data aggregation is that eliminates redundant data transmission and enhances the lifetime of energy in MWSN. In this paper, Author propose, analyze and validate efficient cluster head selection scheme in Mobile Data Collector based routing protocol for data aggregation, which is based on multi-hop routing strategy. Moreover, our approach is better than traditional LEACH in terms of energy consumption of sensor nodes and enhances the network lifetime due to less energy consumption during data transmission.

Akyildiz, I.F et al [12] "A survey on sensor networks" describes advancement in wireless communications and electronics has enabled the development of low-cost sensor networks. The sensor networks can be used for various application areas (e.g., health, military, home). For different application areas, there are different technical issues that researchers are currently resolving. The current state of the art of sensor networks is captured in this article, where solutions are discussed under their related protocol stack layer sections. This article also points out the open research issues and intends to spark new interests and developments in this field.

Arshad, M et al [18] "Routing strategies in hierarchical cluster based mobile wireless sensor networks" Ubiquitous communication networks is a keystone for New Generation Network (NWGN). Mobile Wireless Communication Networks (MWSN) is a viable solution to accomplish the requirements of NWGN. This paper enlightens a comprehensive comparison between single and multi hop inter-cluster routing strategy from cluster head to base station. Moreover, the performance of multi hop routing is calculated and compared with single hop LEACH routing strategy. The simulation results reveal that multi hop routing strategy is to increase the sensor nodes throughput and network lifetime but not efficient approach for delay sensitive and data reliable applications.

Qin Wang; Hempstead et al [11] "A Realistic Power Consumption Model for Wireless Sensor Network Devices" describes realistic power consumption model of wireless communication subsystems typically used in many sensor network node devices is presented. Simple power consumption models for major components are individually identified, and the effective transmission range of a sensor node is modeled by the output power of the transmitting power amplifier, sensitivity of the receiving low noise amplifier, and RF environment. Using this basic model, conditions for minimum sensor network power consumption are derived for communication of sensor data from a source device to a destination node.

Amundson, I et al [7] "Mobile sensor localization and navigation using RF Doppler shifts" over the past decade, wireless sensor networks have advanced in terms of hardware design, communication protocols, resource efficiency, and other aspects. Recently, there has been growing interest in mobile wireless sensor networks, and several small-profile sensing devices that are able to control their own movement have already been developed. Unfortunately, resource constraints inhibit the use of 


\section{International Journal of Science and Research (IJSR) \\ ISSN (Online): 2319-7064 \\ Index Copernicus Value (2013): 6.14 | Impact Factor (2014): 5.611}

traditional navigation methods, because these typically require bulky, expensive, and sophisticated sensors, substantial memory and processor allocation, and a generous power supply.

\section{Problem Formulation}

In Wireless Sensor Networks, existing energy efficient routing algorithms assumed that the sensor nodes are stationary. Some of the applications in WSN must combine with both mobile sensor nodes and fixed sensor nodes in the same networks. In mobile wireless sensor network, sensor nodes relocate themselves with the intention of covering area to be monitored or to replace uncharged or defective sensor nodes. In some situations, sensors are just dropped from a height or thrown parabolic and supposed to position themselves to track something or monitor an area. In such cases, energy becomes a vital issue for sensor nodes as they do not contain any battery recharging facility as well as they are very small in size hence cannot resist high battery capacity. For such an environment, energy needs to be conserved at each step either sensing or processing or communicating or moving. Since the nodes are equipped with a lesser amount of memory, restricted battery power, little computation capability, and small range of communication, the mobility of nodes causes performance degradation. It has been observed that clustering plays an important role in energy saving with better resource allocation.

Many clustering based algorithms are available in mobile wireless sensor networks. In Energy Efficient Cluster Head Selection Protocol in Mobile Wireless Sensor Network (EECHS-MWSN), the cluster-head nodes are selected from the residual energy, lowest mobility factor and density of the node. The proposed study focuses on application of a distributed fault-tolerant clustering algorithm and run time recovery of the sensor nodes from the faulty cluster in mobile wireless sensor networks. This fault can be removed by using back up cluster or by setting the root information as a prefix.

\section{Proposed Methodology}

First Phase: - In the first phase of the proposed work MWSN will be analyzed and developed on the basis of parameters utilized in MWSN. In this scenario number of nodes, routing protocol and energy provided to each and every node will be provided. Mobility model for sensor nodes will be defined in this phase. Each and every node will be provided a deployment location and according to mobility model these nodes will sense information from the particular area.

Second phase- In this phase the scenario will be decomposed into different clusters on the basis of similarity between sensor nodes. Cluster head selection will be done in this phase on the basis of energy available in a single cluster.

Third Phase- In this phase different simulations will be performed using on demand routing protocol for data transmission to base station and a recovery node will be developed that will retrieve information throughout the network. Recovery node is a fault tolerance approach from which data can be recovered if any node or cluster head undergoes into failure.

\section{Results and Discussions}

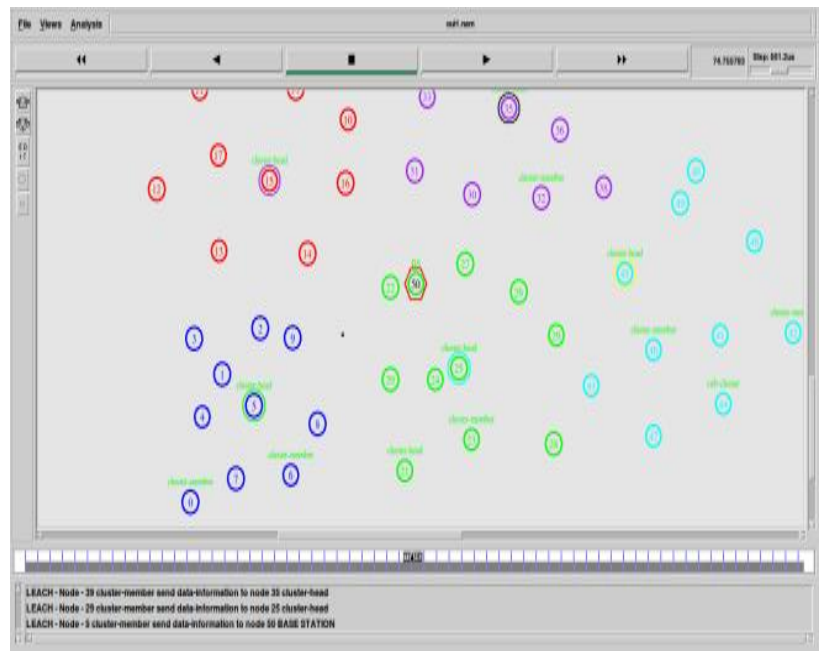

Scenario 6.1: Transmission b/w Cluster Heads \& Base Station

This scenario is used to represent the Transmission of data $\mathrm{b} / \mathrm{w}$ the cluster heads \& the base stations. All the cluster heads send data to the base station i.e node 50 .

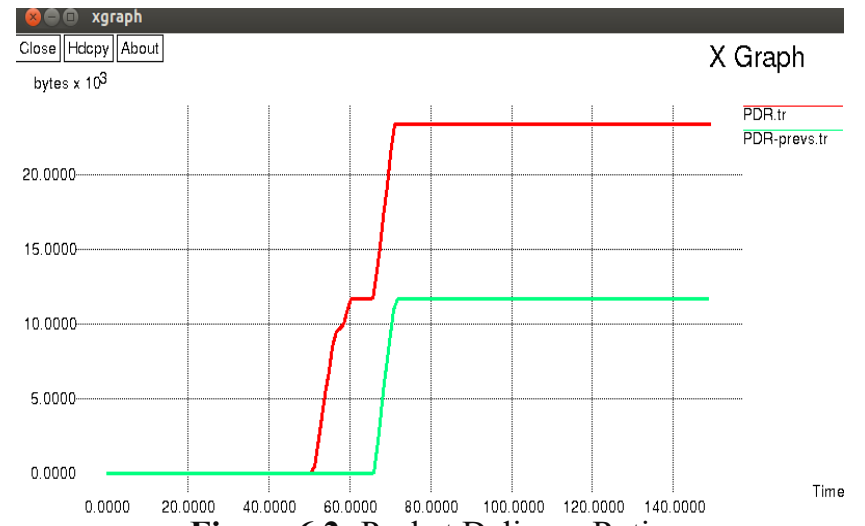

Figure 6.2: Packet Delivery Ratio

It is the ratio of all the received data packets at the destination to the number of data packets sent by all the sources. It is calculated by dividing the number of packet received by destination through the no. of packet originated from the source.

$\mathrm{PDR}=\left(\mathrm{P}_{\mathrm{r}} / \mathrm{P}_{\mathrm{s}}\right) * 100$

Where, $\mathrm{P}_{\mathrm{r}}$ is total packet received and $\mathrm{P}_{\mathrm{s}}$ is total packet sent. 
International Journal of Science and Research (IJSR)

ISSN (Online): 2319-7064

Index Copernicus Value (2013): 6.14 | Impact Factor (2014): 5.611

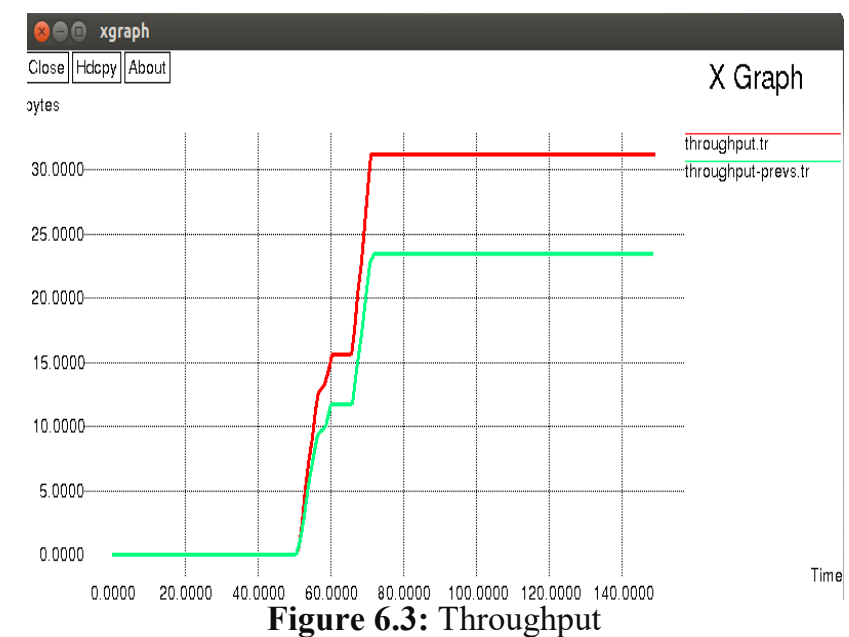

It is the average at which data packet is delivered successfully from one node to another over a communication network. It is usually measured in bits per second.

Throughput $=($ no of delivered packets $*$ packet size $) /$ total duration of simulation.

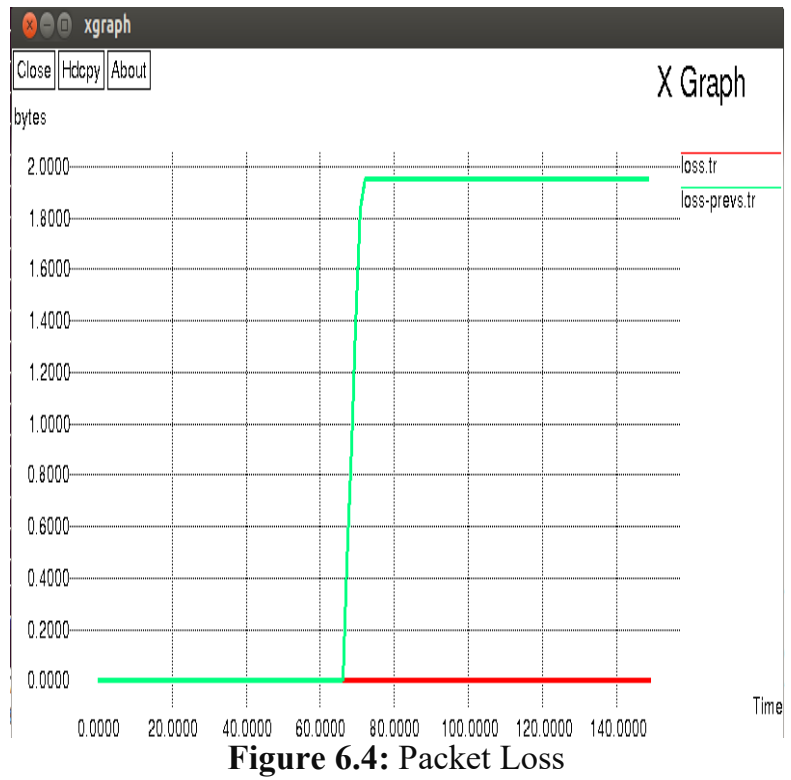

Loss is defined as the number of packet loss when we transfer packets over the network. Packet loss occurs when one or more packets of data travelling across a computer network fail to reach their destination. Packet loss is typically caused by network congestion. Packet loss is measured as a percentage of packets lost with respect to packets sent. The Transmission Control Protocol (TCP) detects packet loss and performs retransmissions to ensure reliable messaging.

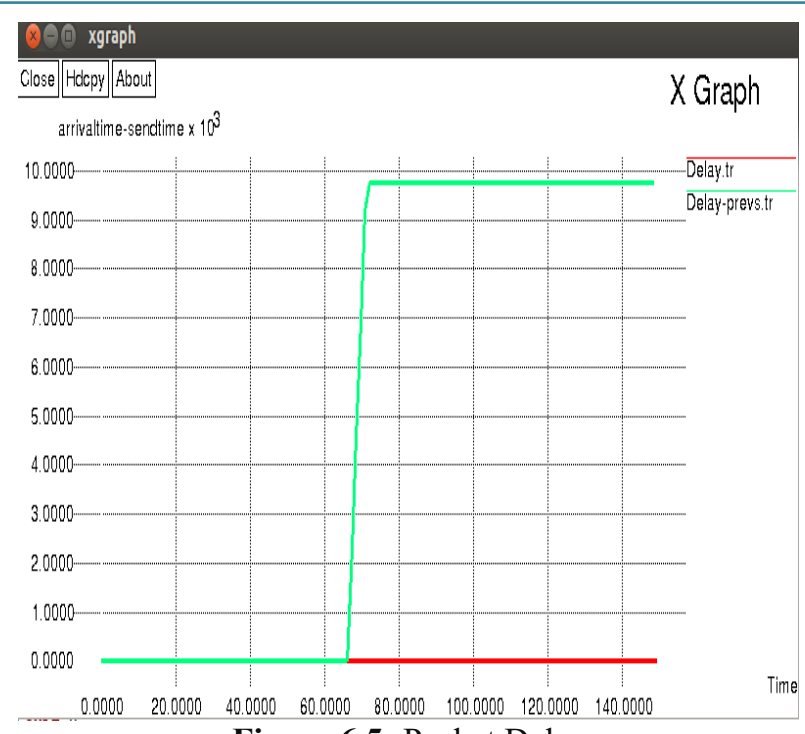

Figure 6.5: Packet Delay

This includes all possible delays caused by buffering during route discovery, latency, and retransmission by intermediate nodes, processing delay and propagation delay. It is calculated as

$$
\mathrm{D}=\left(\mathrm{T}_{\mathrm{r}}-\mathrm{T}_{\mathrm{s}}\right)
$$

Where, $T_{r}$ is receive time and $T_{s}$ is sent time of the packet.

\section{Conclusions}

Wireless sensor network is used for sensing the information by different sensor available in the particular area. In the mobile wireless sensor network different sink nodes have been utilized for sensing the information with mobility. The sink nodes move with a speed in the network to receive the data from various sensor nodes. In the purposed work clustering has been done and cluster head has been selected on the basis of maximum energy containing node. The sensing node transmits the data to the cluster head and cluster head transmit to the base station. The clustering approach increase network lifetime due to each node have chance of cluster head. Proper energy has been utilized. Data recovery has been done at any node failure by using the backup or recovery node. This back up node captured data from all the nodes at a particular interval of time and transmits this data to the base station. Packet delivery ratio, packet loss, end to end delays and throughput has been analyzed for performance evaluation. One the basis of these parameters one can conclude that purposed work provides better results than previous approach.

In the future reference Sink nodes can be utilized with mobility \& sensors nodes with static location for energy optimization of wireless sensor network. Second optimization approach can be utilized for discovery of short route for data transmission so that minimum energy will be consumed by the network.

\section{References}

[1] Yong-Sik Choi "A study on sensor nodes attestation protocol in a Wireless Sensor Network", ISSN 978-14244-5427-3, pp 1738-9445, IEEE, 2010. 
[2] Yuling Lei, "The Research of Coverage Problems in Wireless Sensor Network", ISSN 978-0-7695-3901-0, pp 31 - 34, IEEE, 2009.

[3] Mittal, R. "Wireless sensor networks for monitoring the environmental activities" 978-1-4244-5965-0, pp. 1 - 5, IEEE, 2010.

[4] Yong-Sik Choi "A study on sensor nodes attestation protocol in a Wireless Sensor Network", 978-1-42445427-3, 1738-9445, IEEE, 2010.

[5] Yuling Lei, "The Research of Coverage Problems in Wireless Sensor Network", 978-0-7695-3901-0, pp. 31 - 34, IEEE, 2009.

[6] Mittal, R., "Wireless sensor networks for monitoring the environmental activities", 978-1-4244-5965-0, 1 5, IEEE, 2010.

[7] Amundson, I., Koutsoukos, X., Sallai, J. "Mobile sensor localization and navigation using RF doppler shifts," In: 1st ACM International Workshop on Mobile Entity Localization and Tracking in GPS-less Environments, MELT (2008).

[8] Xuhui Chen, "Research on hierarchical mobile wireless sensor network architecture with mobile sensor nodes", ISSN 978-1-4244-6495-1, pp 2863 2867, IEEE, 2010.

[9] M.Contia "Clone wars: Distributed detection of clone attacks in mobile WSNs", 4321 6754, 123-543, IEEE, 2013.

[10] Xuhui Chen "Research on hierarchical mobile wireless sensor network architecture with mobile sensor nodes", 978-1-4244-6495-1, pp. 2863 - 2867, IEEE, 2010.

[11] Qin Wang; Hempstead, M.; Yang, W.; "A Realistic Power Consumption Model for Wireless Sensor Network Devices," Sensor and Ad Hoc Communications and Networks, 3rd Annual IEEE Communications Society on, vol.1, no., pp.286-295, 28-28 Sept. 2006.

[12] Akyildiz, I.F “A survey on sensor networks", ISSN 0163-6804, pp 102 - 114, IEEE, 2002.

[13] Muhammad Arshad1 "Efficient Cluster Head Selection Scheme in Mobile Data Collector Based Routing Protocol", ISSN 978-1-4577-1967-7, IEEE, 2011.

[14] Arshad, M.; Saad, N.M.; Kamel, N.; Armi, N.; "Routing strategies in hierarchical cluster based mobile wireless sensor networks," International Conference on Electrical, Control and Computer Engineering (INECCE), 2011, vol., no., pp.65-69, 21-22 June 2011.

[15] Md Azharuddin "A Distributed Fault-tolerant Clustering Algorithm for Wireless Sensor Networks", ISSN 978-1-4673-6217-7, IEEE, 2013.

[16] Md Azharuddin "A Distributed Fault-tolerant Clustering Algorithm for Wireless Sensor Networks", 978-1-4673-6217-7, IEEE, 2013.

[17] Deshpande, V.V. "Energy efficient clustering in wireless sensor network using cluster of cluster heads", 978-1-4673-5997-9, 2151-7681, IEEE, 2013.

[18] Vithya, G. "Actuation sensor with adaptive routing and QOS aware checkpoint arrangement on Wireless Multimedia Sensor Network", 978-1-4577-0588-5, 444 - 449, IEEE, 2011. 